\title{
The Complex Model Of The Universe
}

\author{
Dr. Narayan Kumar. Bhadra \\ Lakshmipur Swamiji Seva Sangh High School Laskhmipur, Gobardanga, 24 Parganas(N), West \\ Bengal, India
}

\begin{abstract}
We study a super unified theory with gauge group $S U(11)$. The subgroup $S U(6)$ has been interpreted as a new type of energy source other than $S U(5)[S U(5) \supset S U(3) \times S U(2) \times U(1)]$, where $S U(3)$ the strong energy group; $S U(2)$ the weak energy group \& $U(1)$ the electro dynamics]. We consider a $(4+D)$-dimensional Friedmann-Robertson-Walker type universe having complex scale factor $R+i R_{I}$, where $R$ is the scale factor corresponding to the usual 4-dimensional Universe while $R_{I}$ is that of D-dimensional space. It is then compared with $(4+D)$-dimensional Kaluza-Klein Cosmology having two scale factors $R$ and $a\left(=i R_{I}\right)$. It is shown that the rate of compactification of higher dimension depends on extra dimension ' $D$ '. The Wheeler-DeWitt equation is constructed and general solution is obtained. It is found that for $D=6$ (i.e. in 10 dimension), the Wheeler-DeWitt equation is symmetric under the exchange $R_{I} \leftrightarrow R$.
\end{abstract}

\section{Introduction}

One of the exciting discoveries of $20^{\text {th }}$ century is the unified theory of fields. A natural query is what happened before unification-may be called super unified field.

The question of why the large energy is not found in practical, the observed vacuum energy is so small in comparison to the scales of particle physics is known as cosmological constant problem. It is generally thought to be easier to imagine an unknown mechanism which would set vacuum parameter exactly to zero and so it can be considered that there exist another unification in the very early universe. This class of symmetry group can be expressed mathematically as SU(11) $\supset$ $\mathrm{SU}(5) \times \mathrm{SU}(6) \times \mathrm{U}(1)$. Before the explanation of the groups $\mathrm{SU}(11), \mathrm{SU}(6)$ and $\mathrm{SU}(5)$, we give an example, such as, the change of state of a matter, where it is required latent heat, but this latent heat can not be measured by any instrument but its existence can not be ignored at any cost. As such when in the liquid state the energy of the group $\mathrm{SU}(5)$ changes to the ultimate reality of the group of energy $\mathrm{SU}(11)$, in the vapor state, there must exists another type of energy group SU(6) like latent heat, its name may be given as 'intelligence', which are responsible for the acceleration of the energy of SU(5). and also the cause for the creation of D. N. A. of the live body etc.

After the separation of two type energies SU(5) \& SU(6), they want to interact each other and has a tendency to unify once again, as a result the direction of the energy group SU(6) is opposite to the direction of the energy group SU(5), remaining the temperature unaltered and then an inflation occurred instantaneously. After then, the latent energy SU(6) acts as a field.. In particular the decoupled of SU(11), gave a several domain compartment along with the domain wall in which the compartment filled either latent energy or matter energy and hence created an inhomogeneous discrete energy universe. Then the latent energy compartments together form a core of a massive universe, while the matter energy compartments together form the surface of the universe. As the total number of bosons of $\mathrm{SU}(6)$ is greater than the total number of bosons of $\mathrm{SU}(5)$, so that latent energy of $\mathrm{SU}(6)$ stayed in the centre of the massive universe and form $\mathrm{PBH}$, while the matter energy of SU(5), laid out side the centre region of the early universe and hence produce event horizon by the energy group $\mathrm{SU}(6)$ and particle horizon by the energy group $\mathrm{SU}(5)$. The energy group SU(11) do not interact with the energy group SU(6) and SU(5).

\section{Intelligence: $\mathbf{S U}(6)$}

In the transformations under the group $\mathrm{SU}(6)$, the basic fields here are the latent energy field and we have $\quad \mathrm{U}=\exp (-\mathrm{iH}) \ldots \ldots \ldots$ (1). Where $\mathrm{H}$ is a $6 \times 6$ Hermitian matrix of Zero trace. We have 35 matrix charges $I_{1}, I_{2}, I_{3}, \ldots \ldots \ldots . I_{35}$ out of which five matrices are diagonal. Corresponding to this, we have 35 bosons. For want of any specific designation, they are referred to simply as $J_{k}$. We expect the participating interactions of the bosons $J_{k}$ to have comparable strength. The $\mathrm{J}_{\mathrm{k}}$ bosons are expected to generate a latent force. This force is believed to be 
potentially so large that the exotic matter fluid are expected to transfer into the ordinary matter and then everything of the universe.

\section{Super Unified Field : SU(11)}

If we wish to unify all three interactions $\mathrm{SU}(6), \mathrm{SU}(5), \mathrm{U}(1)$ in a Super Grand Unification scheme, we could trivially combine the three into a structure

$$
\mathrm{SU}(6) \times \mathrm{SU}(5) \times \mathrm{U}(1)
$$

From the symmetry breaking of SU(11), we find $\mathrm{SU}(6)$ and $\mathrm{SU}(5)$; the subgroups of $\mathrm{SU}(11)$, where $\mathrm{p}(=5)>1, \mathrm{n}-\mathrm{p}(=11-5=6)>1$

So that $\mathrm{SU}(\mathrm{n}) \supset \mathrm{SU}(\mathrm{p}) \times \mathrm{SU}(\mathrm{n}-\mathrm{p}) \times \mathrm{U}(1)$

Or, $\quad \mathrm{SU}(11) \supset \mathrm{SU}(5) \times \mathrm{SU}(6) \times \mathrm{U}(1)$

Since the rank of $S U(11)$ is 10 and $U(1)$ is 1 , a useful check is that the sum of the ranks of the subgroup SU(5) and SU(6) is less than or equal to the rank of the original group.

Thus, however, it was realized that such a structure (2) can form part of a single larger structure denoted by SU(11). Again, if we go back to equation (1) and apply it to $11 \times 11$ matrices, the matrix $\mathrm{H}$ has 120 arbitrary constant. Thus there are 120 bosons that now mediate between the different type of energy fields. Of these we already have 24 from SU(5) and 35 from SU(6) and 1 from $\mathrm{U}(1)$. Thus, $120-(24+35+1)=60$ more bosons are needed to make up the list of 120 . For want of any specific designation, they are referred to simply as the $\mathrm{J}$ bosons. The $\mathrm{J}$ bosons are expected to link the participants of $\mathrm{SU}(6)$ with $\mathrm{SU}(5)$ i.e. $\mathrm{SU}(2)$ \& $\mathrm{SU}(3)$ and $\mathrm{U}(1)$. There are emitted and absorbed particles (an anti-J particles). It can be expected, that for the symmetry breaking of SU(11), created an amount of positive energy, negative energy and an equivalent amount of latent energy.

In the SU(11) theory, therefore it is possible to change any of 30(thirty) latent energy bosons into any of the 30(thirty) matter energy bosons or vice-versa by the exchange of the Jbosons. That is why it becomes possible to create or destroy matter particles and hence the universe seem to appear from "nothing".

Again, if we go out-side the gravitating sphere, we see the gravitation would be weaker and weaker. According to Einstein's general relativity, the matter-space-time cannot be separated by any cost. Thus, out-side the Einstein's universe, where real time can not be defined, so the corresponding space (although, the matter belongs to another phase) must be measured as imaginary. Thus the space-time of the universe is actually a complex space-time. Here we consider the real space-time (i.e. unfolded) for Einstein and imaginary space-time (i.e. folded) for us. We find a relation between folded and unfolded space-time of the universe by using Wheeler De-Witt equation.

\section{Source of Background Radiation :}

In the super-unified theory, whenever there is a breakdown of SU(11) into a sub group like $\mathrm{SU}(5) \times \mathrm{SU}(6) \times \mathrm{U}(1)$ which contain the $\mathrm{U}(1)$ group, there inevitable arise particles that have the characteristics of a magnetic monopole. Monopoles are highly stable particles and once created they are not destructible. And so they would survive as relics to the present epoch. Hence we conclude the background radiation are 'primordial' have arisen from discrete sources and got a powerful energy from the energy group $\mathrm{SU}(6)$.

\section{Cosmology with complex scale factor:}

Consider the metric in which the space-time is assumed to be of Robertson-Walker type having a complex scale factor $\mathrm{R}+\mathrm{iR}_{\mathrm{I}}$, where the scale factor $\mathrm{R}$ stands for $(3+1)$ - dimensional space-time and $\operatorname{iR}_{\mathrm{I}}(=\mathrm{a})$ is that for the internal space with dimension $\mathrm{D}$. Avoiding the imaginary term, the line element can be expressed in the form

$$
d s^{2}=-N^{2}(t) d t^{2}+R^{2}(t) \frac{d x^{i} d x^{j}}{\left(1+\frac{k r^{2}}{4}\right)^{2}}+a^{2}(t) \frac{d y^{a} d y^{b}}{\left(1+k^{\prime} \rho^{2}\right)^{2}}
$$

where $\mathrm{N}(\mathrm{t})$ is the lapse function, $\mathrm{r}^{2}=\mathrm{x}^{\mathrm{i}} \mathrm{x}^{\mathrm{i}}(\mathrm{i}=1,2,3), \rho^{2}=\mathrm{y}^{\mathrm{a}} \mathrm{y}^{\mathrm{a}}(\mathrm{a}=1,2,3, \ldots \ldots, \mathrm{D})$ and $\mathrm{k}, \mathrm{k}^{\prime}=0$, \pm 1 , for flat, closed or open type of 4-dimensional universe and $\mathrm{D}$-dimensional space. For simplicity, we assume the internal space to be flat i.e. $\mathrm{k}^{\prime}=0$. The form of energy-momentum tensor is chosen as $T_{A B}=\left(-\rho, p, p, p, p_{D}, p_{D}, \ldots \ldots \ldots \ldots \ldots, p_{D}\right) \ldots \ldots \ldots \ldots . .(5)$. Now, we examine the case for which the pressure along all the extra-dimensions vanishes, namely, $\mathrm{p}_{\mathrm{D}}=0$. In doing so, we are 
motivated by the brane world scenarios where the matter is to be confined to the 4-dimensional universe, i.e. auxiliary hyper-space, so that all components of $\mathrm{T}_{\mathrm{AB}}$ is set to zero but the space-time components and it means no matter escapes through the extra dimensions. We assume the energymomentum tensor $\mathrm{T}_{\mu \nu}$ to be that of an exotic $\chi$ fluid with the equation of state

$$
\mathbf{p}_{\chi}=\left(\frac{\mathbf{m}}{\mathbf{3}}-\mathbf{1}\right) \rho_{\chi}
$$

Where $\mathrm{p}_{\chi}$ and $\rho_{\chi}$ are the pressure and density of the fluid, respectively and the parameter $\mathrm{m}$ is restricted to the range $0 \leq \mathrm{m} \leq 2$ so that there is violation of strong energy condition and the universe experiences accelerated expansion. The scalar curvature corresponding to the metric (4) has the expression

$$
R=\frac{-6 R R_{I} N \ddot{R}+6 R R_{I} \dot{N} \dot{R}^{2}-2 R^{2} \ddot{R}_{I}^{9} N+2 R^{2} \dot{N}_{I}-2 R_{I} N^{3} k+R_{I} N^{3} k^{2} r^{2}-6 R_{I} N \dot{R}^{2}-6 R \dot{R}_{I} N}{R^{2} N^{3} R_{I}}
$$

Now substituting it into the dimensionally extended Einstein-Hilbert action (without higher dimensional cosmological term) including a matter term indicating the above mentioned exotic fluid the effective Lagrangian becomes

$$
\begin{aligned}
L=i^{\mathrm{D}}\left[\frac{1}{2 \mathrm{~N}} R \mathbf{R}_{\mathrm{I}}^{\mathrm{D}} \dot{\mathbf{R}}^{2}+\right. & \frac{\mathrm{D}(\mathrm{D}-1)}{12 \mathrm{~N}} \mathbf{R} \mathbf{R}_{\mathrm{I}}^{\mathrm{D}-2} \dot{R}_{\mathrm{I}}^{2}+\frac{\mathrm{D}}{2 \mathrm{~N}} \mathbf{R}^{2} \mathbf{R}_{\mathrm{I}}^{\mathrm{D}-1} \dot{\mathbf{R}} \dot{R}_{\mathrm{I}} \\
& \left.-\frac{1}{2} k N R R_{\mathrm{I}}^{\mathrm{D}}+\frac{1}{6} \mathrm{~N} \rho_{\chi} \mathbf{R}^{3} \mathbf{R}_{\mathrm{I}}^{\mathrm{D}}\right]
\end{aligned}
$$

The continuity equation, by using the contracted Bianchi identity in (4+D) dimensions, namely

$\nabla_{\mathrm{M}}^{\mathrm{G}^{\mathrm{MN}}}=\nabla_{\mathrm{M}}^{\mathrm{T}^{\mathrm{MN}}}=0 \ldots \ldots$. (9) together with the assumption that the matter is confined to $(3+1)$ dimensional space-time gives $\nabla_{\mathrm{i}} \mathrm{T}^{\mathrm{ij}}=0 \quad$ i.e. $\dot{\boldsymbol{\rho}}_{\chi} \mathbf{R}+\mathbf{3}\left(\mathbf{p}_{\chi}+\boldsymbol{\rho}_{\chi}\right) \dot{\mathbf{R}}=\mathbf{0} \ldots$ (10).

Using (6) into the continuity equation $(10)$, the energy density in a closed $(\mathrm{k}=1)$ FriedmannRobertson-Walker universe is

$$
\rho_{\chi}(\mathbf{R})=\rho_{\chi}\left(\mathbf{R}_{\mathbf{o}}\right)\left(\frac{\mathbf{R}_{\mathbf{o}}}{\mathbf{R}}\right)^{\mathrm{m}}
$$

reference time $t_{0}$.

where $R_{o}$ is the value of $R$ at an arbitrary

Again, if we believe that the cosmological term plays an important role in vacuum energy density, then we may the cosmological term as $\quad \Lambda \equiv \rho_{\chi}(\mathrm{R})$.

With

$$
\Lambda(\mathbf{R})=\Lambda\left(\mathbf{R}_{\mathbf{o}}\right)\left(\frac{\mathbf{R}_{\mathbf{0}}}{\mathbf{R}}\right)^{\mathrm{m}} \quad{ }^{\text {i.e. }} \mathbf{L}=\mathbf{i}^{\mathrm{D}} \mid \frac{1}{2 \mathbf{N}} \mathbf{R} \mathbf{R}_{\mathrm{I}}^{\mathrm{D}} \dot{\mathbf{R}}^{2}+\frac{\mathbf{D}(\mathbf{D}-\mathbf{1})}{12 \mathrm{~N}} \mathbf{R}^{3} \mathbf{R}_{\mathrm{I}}^{\mathrm{D}-2} \dot{\mathbf{R}}_{\mathrm{I}}^{2}+\frac{\mathrm{D}}{2 \mathbf{N}} \mathbf{R}^{2} \mathbf{R}_{\mathrm{I}}^{\mathrm{D}-1} \dot{\mathbf{R}} \dot{\mathbf{R}}_{\mathrm{I}}
$$

Taking $\mathrm{m}=2$ and

$$
\Lambda(\mathrm{R})=\frac{3}{\mathrm{R}^{2}}
$$

We have

The lapse function $\mathrm{N}(\mathrm{t})$, is an arbitrary function of time due to the fact that Einstein's general relativity is a reparametrization invariant theory. We therefore, take the gauge

$$
\mathbf{N}(\mathbf{t})=\mathbf{R}^{\mathbf{3}}(\mathbf{t}) \mathbf{R}_{\mathbf{I}}^{\mathbf{D}}(\mathbf{t}) \mathbf{i}^{\mathbf{D}} \quad \text { (15) and then Lagrangian (12) becomes }
$$

$$
\mathbf{L}=\frac{1}{2} \frac{\dot{\mathrm{R}}^{2}}{\mathbf{R}^{2}}+\frac{\mathrm{D}(\mathrm{D}-1)}{12} \frac{\dot{\mathrm{R}}_{\mathrm{I}}^{2}}{\mathbf{R}_{\mathrm{I}}^{2}}+\frac{\mathrm{D}}{2} \frac{\dot{\mathbf{R}}}{\mathbf{R}} \frac{\dot{\mathrm{R}}_{\mathrm{I}}}{\mathbf{R}_{\mathrm{I}}}
$$


We now define the new variables Then the equation (16) becomes

The equation of motion are obtained

$$
\mathrm{X}=\log \mathrm{R}, \mathrm{Y}=\log \mathrm{R}_{\mathrm{I}}
$$

$$
\mathbf{L}=\frac{1}{2} \dot{\mathbf{X}}^{2}+\frac{\mathrm{D}(\mathrm{D}-1)}{12} \dot{\mathbf{Y}}^{2}+\frac{\mathrm{D}}{2} \dot{\mathbf{X}} \dot{\mathbf{Y}}
$$

$$
\begin{aligned}
& \ddot{\mathbf{X}}+\frac{\mathrm{D}}{2} \ddot{\mathbf{Y}}=0 \\
& \ddot{\mathbf{X}}+\frac{\mathrm{D}-1}{3} \ddot{\mathbf{Y}}=\mathbf{0}
\end{aligned}
$$

Solving equations (19) and (20) we get,

$$
\ddot{\mathbf{X}}=0 \ldots \ldots \ldots \ldots(21) \text { and } \quad \ddot{\mathbf{Y}}=\mathbf{0}
$$

The solutions for $\mathrm{X}$ and $\mathrm{Y}$ from equations (21) and (22) are

$$
\begin{array}{rll} 
& \mathrm{X}=\mathrm{At}+\gamma, \quad \mathrm{Y}=\mathrm{Bt}+\delta \\
\therefore \quad & \mathrm{R}(\mathrm{t})=\mathrm{A}^{\prime} \mathrm{e}^{\alpha \mathrm{t}} \quad \text { and } \quad \mathrm{R}_{\mathrm{I}}(\mathrm{t})=\mathrm{B}^{\prime} \mathrm{e}^{\beta \mathrm{t}}
\end{array}
$$

where the constants $\mathrm{A}, \mathrm{B}, \gamma$ and $\delta$ or $\mathrm{A}^{\prime}, \mathrm{B}^{\prime}, \alpha \& \beta$ should be obtained from the initial conditions. Consider the size of all spatial dimensions be the same at $t=0$ and assumed that this size would be the planck size $\ell_{\mathrm{p}}$ in accordance with quantum cosmological considerations. So we take $\mathrm{R}(0)=\mathrm{R}_{\mathrm{I}}(0)$ $=\ell_{\mathrm{p}}$ so that $\mathrm{A}^{\prime}=\mathrm{B}^{\prime}=\ell_{\mathrm{p}}$. Thus $\mathrm{R}(\mathrm{t})=\ell_{\mathrm{p}} \mathrm{e}^{\alpha \mathrm{t}}$ and $\mathrm{R}_{\mathrm{I}}(\mathrm{t})=\ell_{\mathrm{p}} \mathrm{e}^{\beta \mathrm{t}}$ It is important to note that the constants $\alpha, \beta$ are not independent and a relation may be obtained between them. This is done by imposing the zero energy condition $\mathrm{H}=0$ which is the well-known result in cosmology due to the existence of arbitrary laps function $\mathrm{N}(\mathrm{t})$ in the theory. The Hamiltonian constraint is obtained through the legender transformation of the Lagrangian (18)

$$
\begin{array}{r}
\left.H=\frac{1}{2} \dot{X}^{2}+\frac{D(D-1}{12}\right) \dot{Y}^{2}+\frac{D}{2} \dot{X} \dot{Y}=0 \\
\text { i.e. } \quad H=\frac{1}{2} \alpha^{2}+\frac{D(D-1)}{12} \beta^{2}+\frac{D}{2} \alpha \beta=0
\end{array}
$$

This constraint is satisfied only for $\alpha \leq 0, \quad \beta \geq 0$ or $\alpha \geq 0, \beta \leq 0$

$o$ find $\alpha, \beta$, we first obtained the Hubble parameter for $\mathrm{R}(\mathrm{t})$. Again,

$\mathbf{H}_{\mathrm{I}}=\frac{\dot{\mathbf{R}}_{\mathrm{I}}}{\mathbf{R}_{\mathrm{I}}} \boldsymbol{\beta} \quad \mathbf{H}_{\mathbf{R}}=\frac{\dot{\mathbf{R}}}{\mathbf{R}}=\alpha \quad$ and the Hubble parameter which the
constant $\alpha$ is fixed. $\therefore \quad \mathrm{R}(\mathrm{t})=\ell_{\mathrm{p}} \mathrm{e}^{\mathrm{H}_{\mathrm{R}} \mathrm{t}}, \quad \mathrm{R}_{\mathrm{I}}(\mathrm{t})=\ell_{\mathrm{p}} \mathrm{e}^{-\mathrm{H}_{\mathrm{R}} \mathrm{t}}$

for $\mathrm{D}=1$ also, if $\alpha \neq 0$, then $\alpha=-\beta$ or $\alpha+\beta=0$, or

$\mathbf{X}+\mathbf{Y}=\mathbf{0}=\mathbf{X}+\mathrm{Y}=$ constant, or $\log \mathrm{R}+\log \mathrm{R}_{\mathrm{I}}=$ constant, $\therefore \mathrm{RR}_{\mathrm{I}}=$ constant. Again, for $\mathrm{D} \neq 1$, then either $\alpha=0$ or $\beta=0$ i.e. $\dot{\mathbf{X}}=0$ or,$\dot{\mathbf{Y}}=\mathbf{0} \therefore \quad \mathrm{X}=$ constant, $\quad \mathrm{Y}=$ constant.

$\mathrm{R}=\mathrm{R}_{\mathrm{I}}=\ell_{\mathrm{p}}$ (a time independent scale factor) Therefore, for $\mathrm{D}>1$,

So

$$
\alpha_{ \pm}=\frac{D B}{2}\left[-1 \pm \sqrt{1-\frac{2}{3}\left(1-\frac{1}{D}\right)}\right]
$$

i.e.

$$
\mathrm{H}_{\mathrm{R}^{ \pm}}=\frac{\mathrm{DH}_{\mathrm{I}}}{2}\left[-1 \pm \sqrt{1-\frac{2}{3}\left(1-\frac{1}{\mathrm{D}}\right)}\right]
$$

$$
\mathrm{R}_{\mathrm{I}}(\mathrm{t})=\ell_{\mathrm{p}} \mathrm{e}^{\mathrm{H}_{\mathrm{I}} \mathrm{t}}
$$

$$
R_{ \pm}(t)=\ell_{p} e^{\frac{D H_{1} t}{2}}-1 \pm \sqrt{1-\frac{2}{3}\left(1-\frac{1}{D}\right)}
$$


and

$$
\begin{aligned}
& \mathbf{R}(\mathbf{t})=\ell_{\mathrm{p}} \mathrm{e}^{\mathrm{H}_{\mathrm{R}} \mathrm{t}} \\
& R_{I_{ \pm}}(t)=\ell_{p} e^{\frac{2 \mathrm{HR} t}{D}}\left[-1 \pm \sqrt{1-\frac{2}{3}\left(1-\frac{1}{D}\right)}\right]^{-1}
\end{aligned}
$$

It is easy to show that the Lagrangian (18) (or the equations of motions) is invariant under the transformation $\mathrm{R} \rightarrow \mathrm{R}^{-1}, \quad \mathrm{R}_{\mathrm{I}} \rightarrow \quad \mathrm{R}_{\mathrm{H}}^{-1 \ldots \ldots \ldots}$ (29) Which is consistent with the time reversal $\mathrm{t} \rightarrow-\mathrm{t}$ Therefore we have dynamical symmetry between $R$ and $R_{I}$, namely $R_{I} \leftrightarrow R$

\section{Quantum cosmology over complex space :}

The goal of quantum cosmology by solving the WDW equation over the complex space ( $R$ $\left.+\mathrm{iR}_{\mathrm{I}}\right)$ is to understand the origin and evolution of the universe. In principle, it is very difficult to solve the WDW equation in the super space due to the large number of degrees of freedom. In practice, one has to freeze out of all but a finite number of degree of freedom of the gravitational and matter fields. This procedure is known as quantization in mini-super-space, and will be used in the following. The mini-super-space in our model is two dimensional with gravitational variables $\mathrm{X}$ and $\mathrm{Y}$. To obtain the Wheller-DeWitt equation, in this mini-super-space, we start with the Lagrangian (21). The conjugate momenta corresponding to $\mathrm{X}$ and $\mathrm{Y}$ are obtained as

$\&$

$$
P_{x}=\frac{\partial L}{\partial \dot{X}}=\dot{X}+\frac{D}{2} \dot{\mathbf{Y}} \quad P_{y}=\frac{\partial L}{\partial \dot{\mathbf{Y}}}=\frac{D}{2} \dot{\mathbf{X}}+\frac{D(D-1)}{6} \dot{\mathbf{Y}}
$$

$$
\dot{\mathbf{X}}=\frac{\mathbf{6}}{\mathbf{D}+\mathbf{2}}\left[\mathbf{P}_{x}\left(\frac{\mathbf{1}-\mathbf{D}}{\mathbf{3}}\right)+\mathbf{P}_{\mathrm{s}}\right] \quad \ldots . . . . .(31) \quad \& \quad \dot{\mathbf{Y}}=\frac{\mathbf{6}}{\mathbf{D}(\mathbf{D}-\mathbf{1})}\left[\mathbf{P}_{\mathrm{x}} \frac{\mathbf{2}(\mathbf{1}-\mathbf{D})}{\mathbf{D}+\mathbf{2}}-\mathbf{P}_{x} \frac{\mathbf{D}(\mathbf{1}-\mathbf{D})}{\mathbf{D}+\mathbf{2}}\right]
$$

$$
\begin{aligned}
& \text { Substituting equations (31) \& (32) into the Hamiltonian constraint (23) which obtained through the legender } \\
& \text { transformation of Lagrangian (18) as } \quad \mathbf{H}=(1-D) \mathbf{P}_{x}^{2}-\frac{6}{D} \mathbf{P}_{\mathrm{s}}^{2}+6 \mathbf{P}_{x} \mathbf{P}_{\mathrm{s}}=\mathbf{0} \\
& \text { Now, we may use the following quantum mechanical replacement } \mathbf{P}_{x} \rightarrow-\mathbf{i} \frac{\partial}{\partial \mathbf{X}}, \quad \quad \mathbf{P}_{\Sigma} \rightarrow-i \frac{\partial}{\partial \mathbf{Y}} \\
& \text { and Wheeler-DeWitt equations takes the form }\left[(\mathbf{D}-\mathbf{1}) \frac{\partial^{2}}{\partial \mathbf{X}^{2}}+\frac{6}{\mathbf{D}} \frac{\vec{\partial}^{2}}{\partial \mathbf{Y}^{2}}-\mathbf{6} \frac{\boldsymbol{\partial}}{\partial \mathbf{X}} \frac{\boldsymbol{\partial}}{\boldsymbol{\partial} \mathbf{Y}}\right] \boldsymbol{\Psi}(\mathbf{X}, \mathbf{Y})=\mathbf{0} \quad \text { (34) } \\
& \text { Where } \psi(X, Y) \text { is the wave function of the universe in the }(X, Y) \text { mini-super-space. The general solution is }
\end{aligned}
$$

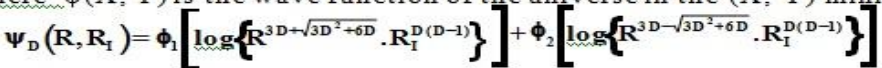

$$
\begin{aligned}
& \text { Using separation of variables } \psi(\mathrm{X}, \mathrm{Y})=\phi(\mathrm{X}) \cdot \psi(\mathrm{Y}) \text {, } \\
& \begin{array}{l}
\text { We obtain the following equations } \frac{\partial \phi}{\partial X}=\frac{\gamma}{D-1} \phi(X) \quad \ldots . . . . .(37) \& \frac{\partial \psi}{\partial Y}=\frac{\gamma}{3+\sqrt{\frac{3(D+2)}{D}}} \psi(Y) \\
\text { where we assume } \gamma>0 \text {. The solutions of equations (36) \& (37) are }
\end{array} \\
& \phi(X)=e^{ \pm \frac{Y X}{D-1}} \ldots . . . . .(38) \text { and } \psi(Y)=e^{ \pm \frac{y}{3 \sqrt{D}+\sqrt{3(D+2)}}} \text {, thus we may write }
\end{aligned}
$$

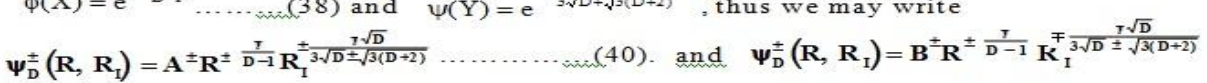

\section{Concluding Remarks:}

So the space-time of the universe is actually a complex space-time and there is neither any starting point nor any ending point of the wider (measurable in quantum cosmology) universe. Only there exists the initial and ending conditions for narrower (measurable in classically) universe, which emerged from wider universe by the process of changing phase, which is a continuous process. So there exists two phases having real space $(R) \&$ pseudo-space $(i R)$ in such a way $R_{I} R_{I}=$ R.R.

\section{Acknowledgement}

The author is thankful to Prof. Subenoy Chakraborty, Department of Mathematics, Jadavpur University for helpful discussion.

\section{References:}

[1] P. J. E. Peebles and B. Ratra, Astrophy. J. Lett. 325 . L17 (1988) : and R. G. Vishwakarma, Class. Quant. Grav. 14. 945(1997).

[2] F. Hoyle. G. Burbidge and J. V. Narlikar, Mon. R. Astron. Soc. 286. 173(1997).

[3] T. A. Appelquist, A. Chodos and P. G. O. Freund, Modern Kaluza-Klein Theories, Frontiers in Physics Series, ( Volume 65), 1986 (Ed. Addison Wesely).

[4] Brian Greene, The Elegant Universe, W.W.Norton\&Company, New York (1999), pp. 357 - 358.

[5] G. Riess et al. Astrophys. J. 560, 49(2001). 